\title{
February 2014 Phoenix Pulmonary Journal Club: Smoking Cessation
}

\author{
Evins AE, Cather C, Pratt SA, et al. Maintenance treatment with varenicline for \\ smoking cessation in patients with schizophrenia and bipolar disorder: a \\ randomized clinical trial. JAMA. 2014;311(2):145-54. [CrossRef] [PubMed]
}

It is estimated that $>50 \%$ of people with mental illness smoke cigarettes. Pharmacotherapy with varenicline has been shown to reach an abstinence rate of $24 \%$ at 12 weeks when compared to placebo at $5 \%$. Once off pharmacotherapy, the maintenance of smoking cessation decreases to $12 \%$ at 6 months. This study examined whether a prolonged maintenance course of varenicline over 40 weeks is associated with increased rates of smoking cessation. The study was a randomized double blind placebo-controlled trial done in 10 community centers. 203 patients met inclusion criteria and entered a 12 week smoking cessation program with varenicline and cognitive behavioral therapy (CBT). 87 patients completed the initial smoking cessation to continue onto the maintenance phase. Patients were randomized to receive either 40 weeks of varenicline and CBT or CBT alone. The results showed that at the end of the 52 week study $60 \%$ (40 patients) maintained smoking abstinence versus $19 \%$ (9 patients) in the placebo group. Side effect profiles were similar in both groups and there were no significant adverse events. The study was well done and showed that varenicline was well tolerated for a prolonged smoking cessation program. The abstinence rate of $60 \%$ at 52 weeks is astonishing and needs to be further validated with larger scale trials. The use of varenicline in patients with psychiatric illness has been met with scrutiny due to its black box warning of increased rates of suicidal ideation and depression. It has been debated that these side effects have been related to smoking cessation rather than drug effect. This study, although small, supports that varenicline can be used provided there is close follow up and continued cognitive behavioral therapy. Larger scale studies are still needed to prove long term efficacy as a smoking cessation maintenance therapy.

\section{Volpp KG, Troxel AB, Pauly MV, et al. A randomized, controlled trial of financial incentives for smoking cessation. N Engl J Med. 2009;360(7):699-709. [CrossRef] [PubMed]}

It is estimated that it costs a company $\$ 3400.00$ a year to employ a smoker versus a nonsmoker. The higher cost is attributed to lower productivity, and absenteeism. This study was a randomized control trial comparing an employer driven smoking cessation utilizing financial incentives to help people quit. The study was performed at a multinational company within the USA. 878 workers were randomized into 2 groups, 442 assigned to control and 436 to the financial incentive group. Both groups received information on smoking cessation programs but the incentive group was also given a financial incentive at the completion of a smoking cessation program (\$100.00), maintenance of smoking cessation for 6 months (\$250.00), and continued maintenance for 1 year (\$450.00). Smoking cessation was confirmed by salivary or a urine cotinine test. The results showed that the incentivized group had higher rates of completing the smoking cessation program ( $20.8 \%$ control vs. $46.3 \%$ treatment ), higher rates of 
cessation at 6 months (11.8\% control vs. 20.9\%) and at 12 months (5\% control vs. $14.7 \%$ treatment). The study did show that financial incentives may contribute to smoking cessation, however there are too many variables that may have confounded the results. The study was done at a large multinational company and targeted a select group of more educated and more motivated individuals.

The average price of pack of cigarettes exceeds $\$ 5$ per day. The average smoker in this study smoked 1 pack per day, The biggest incentive here is the money gained from smoking cessation which is a savings of $\$ 1825.00$ which is far greater than the financial incentive being offered. The cotinine test is only accurate in detecting a certain level of cotinine acquired from smoking usually within the past 7 days. It is possible that the test may have failed to detect mild, intermittent smokers. A policy such as this is a novel concept and has be instituted but in different forms. Rather than a worker receiving a "BONUS" for not smoking, he/she will be required to pay higher premiums for healthcare insurance, or life insurance. Overall this was an interesting study but not practical to apply on a universal scale.

\section{Bullen C, Howe C, Laugesen M, McRobbie H, Parag V, Williman J, Walker N. Electronic cigarettes for smoking cessation: a randomised controlled trial. Lancet. 2013;382(9905):1629-37. [CrossRef] [PubMed]}

Electronic cigarettes have exploded in availability and popularity over the last 5 years. As a nontobacco nicotine delivery device it offers the desirability of tobacco-based smoking without the odor and additional chemicals. The product has been offered as a mechanism to support smoking cessation by many practitioners but the evidence on whether this is prudent is lacking. This study as a large randomized control trial comparing nicotine based e-cigarettes to placebo e-cigarettes and to nicotine patches as a smoking cessation tool. The study was performed in New Zealand. A total of 657 patients were randomized and divided into 3 groups, 289 patients into 13 weeks of ecigarettes with nicotine, 295 patients with nicotine patches, and 73 patients with placebo e-cigarettes. The primary outcome was smoking cessation measured by exhaled carbon monoxide levels at 6 months. The results failed to show a significant difference between the 3 groups. The e-cigarette nicotine group reached cessation in only $7.3 \%$ vs. 5.8\% in the nicotine patch arm and $4.1 \%$ in the placebo e-cigarette arm. The low rates of tobacco cessation were surprising and lower than expected. The authors noted that the study was powered to achieve a $20 \%$ quit rate using nicotine e-cigarettes and because it did not reach this level, there was insufficient evidence to state that e-cigarettes were superior. The low quite rate were puzzling as 5-7\% quite rates have been standard for behavioral therapy alone. The authors concluded that the nicotine content in the ecigarettes may have been to low and also that the first generation of e-cigarettes were less pleasurable. The study was well designed and well done but failed to achieve its primary outcome. E-cigarettes do not have sufficient evidence for us to recommend them as a smoking cessation tool.

Manoj Mathew, MD FCCP MCCM Associate Editor 